\title{
Unity in diversity
}

\section{Gunasekera K D ${ }^{1}$}

Journal of the Ceylon College of Physicians, 2014, 45, 3-5

Immediate Past President Ceylon College of Physicians - Dr. Udaya Ranawake, the Secretary Ministry of Defense and Chairman Urban Development Authority Mr. Gotabhaya Rajapakse - our honored Chief Guest today, distinguished officials from the Ministry of Health, and the WHO, Members of the Council, Past Presidents, Members of the board of Trustees, Fellows and Members of the Collage and distinguished guests.

I am indeed deeply humbled by the honor and trust bestowed on me today by the esteemed Council of the Ceylon College of Physicians. I consider it not a singular honor but also only recognition of the field of Respiratory Medicine which I represent. The last President to hold this prestigious post from my specialty was Dr. C G Uragoda in 1982, my respected mentor, who has graciously accepted our invitation to be here with us today. On behalf of the Ceylon College of Physicians I welcome our Chief guest, the Secretary Ministry of Defense and Chairman Urban Development Authority, Mr. Gotabhaya Rajapakse.

Dr. Udaya Ranawake our immediate past president and his council, have very successfully accomplished the mission of directing the College with excellent academic deliberations, fellowship and International Cooperation during their tenure. I wish to congratulate them on their admirable performance.

Today, I wish to dedicate my Presidential Address to all Physicians - past and present who have contributed and continue to tirelessly contribute providing patient care and good health to all in our country.

I've selected the theme of UNITY IN DIVERSITY for the year 2014. Diversity is a natural strategy, which has ensured the survival and success of the human race. Optimizing on the strengths of diversity by unity is the most powerful tool of success. The field of medicine too has evolved and diversified to meet the ever-increasing demands of health challenges that have threatened human existence.

\footnotetext{
${ }^{1}$ President, Ceylon College of Physicians - 2014. Consultant Respiratory Physician, National Hospital of Sri Lanka.
}

The rapid advances in knowledge, information and technology, diagnostics, therapeutics and communication, have resulted in a vast expansion in the basic fields of medicine, with multiple ramifications. This imposes a great demand on the physician to keep abreast in the modern world. Ready access to medical information has led to a changed profile in the public and patients, who expect the best diagnostic and treatment options from the practicing clinician. Postgraduate training in Medicine has diversified greatly over the past decade. It has catered to the needs of the country by initiating new training programs on par with international standards.

The vast expansion of specialties has resulted in a nationwide network of Consultants throughout the country. This is the strength of the College. This year we hope to enhance regional cooperation and improve the services through physician groups. The College has a representation in the council from each of the provinces in the country this year and thereby hopes to optimise services provided to the regions.

Despite the advances and diversification in the field of medicine a holistic approach to the patient remains of paramount importance. The patient still remains an individual who requires care as a person and hence a multidisciplinary team approach is vital. Close cooperation between the finer specialties and also the general physician is necessary, and I would say essential. The College will encourage the practice of multidisciplinary discussion to be incorporated in patient management as this will lead to improved patient care. To achieve this we envisage representation of the finer specialties in the College Council this year, in order to facilitate, equity of services, research, resource allocation, capacity building and establishment of the newer fields in Medicine.

\section{Changing disease patterns}

In an era of rapid globalization I think it is pertinent to briefly review the global burden of disease, which, we, as Physicians have to address.

With the global picture in mind let us now look at the Sri Lankan scenario and how we have performed in addressing these issues. 


\section{The burden of Non Communicable Disease dominates the picture}

The College working with the Ministry of Health and WHO has taken measures to combat the everimposing challenge of the Non Communicable Diseases. Physicians play an important role as diagnosticians, curative care givers and key opinion leaders in this area. Many outstation educational programs have been organized during the past few years in this respect. As President of the College I pledge my fullest support and commitment to continue the good work done in the past and hope to work with the College of Community Physicians who have done important work in this area to address this national crisis. Amongst the Non Communicable Diseases, Respiratory diseases, my own specialty, contributes to a large burden, but has been sadly neglected and we hope to strengthen this area together with the College of Respiratory Physicians this year.

We cannot, however, be complacent with the unfinished agenda of communicable diseases where constant vigilance needs to be maintained. The College intends to liaise with the National Campaigns of Dengue, TB, HIV, Malaria, Leprosy and Filaria to effectively strengthen disease control and consolidate the gains in these diseases. A big bouquet to the preventive health care system, which has made remarkable achievements. The country has been totally free of malaria transmission for over a year now but new challenges emerge - there is a possibility of delay in diagnosis of imported malaria patients. The problem of dengue rages on, and the resurgence of leprosy causes concern as it was considered eliminated.

\section{Regional cooperation}

Regional cooperation in the sphere of global health is important in the context of similarities in disease patterns geography, socio economic status, culture and ethnicity. Thus far international cooperation of the CCP has predominantly been with the nations of the western hemisphere. Collaboration with the countries in South Asian and the Asia Pacific regions would be a resource of great potential in the context of collaborative research, continued training opportunities for practicing physicians, and sharing knowledge on disease control.

The Asian Network for Obstructive Lung Disease, ANOLD, of which I am a member of the steering committee, is an example of regional cooperation in collaborative research. Through studies conducted over the past 8 years in Badulla and Colombo under my supervision we have provided insight into the Asian Chronic Obstructive Pulmonary Disease profile.

SAARC collaboration in this year's Annual Academic Sessions is envisaged and we would strive to make this a reality in 2014 . I hope to utilize the support from the ANOLD member countries to augment short-term fellowship programs for practicing clinicians in order to introduce and strengthen advances in this field of medicine.

\section{Training and Education}

The College has been heavily committed providing continuous medical education through pathways of seminars, workshops and academic sessions in the past. This year too we hope to conduct many academic activities with special emphasis on skill development through practical hands on workshops with protocols in key areas of clinical relevance.

Strengthening of E-medicine programs, access to online journals and web casting through the College website would be prioritized.

\section{Research}

As a foundation of medicine research is one of the main priorities and hence an important objective of the College. However there are many obstacles to the practicing clinician to engage in research. Restraints on time, finances, secretarial assistance, and lack of incentives and the enormous burden of work have a negative impact on research commitments. Paucity of good national data bases has retarded the development of country-specific guidelines and the effective management of disease.

Some pioneering work has been done in Non Communicable Disease especially in relation to disease burdens of Diabetes and Hypertension. In Respiratory diseases, The Burden of Obstructive Lung Disease (BOLD), The Adult Asthma Survey and the ISAAC - International Study on Asthma and Allergies in Childhood have been national research projects, we carried out to completion recently with international collaboration. Such studies will provide vital information for policy and to plan and develop strategies to combat the high morbidity and mortality of these diseases.

The possibility of providing research assistants, with basic facilities to each clinical unit will be discussed with the $\mathrm{MOH}$ to augment clinical audit and research. We will facilitate the utilization of academic resources of the universities in collaboration with the regional physicians to carry out research in areas of high national priority.

\section{Strategic Plan}

The Ceylon College of Physicians has been nurtured to its current status with much dedication and commitment of many past presidents and Councils. The objectives of the College have stood 
the test of time in providing a framework to maintain the integrity of the complete physician of high caliber. Dr. Udaya Ranawake arduously dedicated himself to formulating the Long Term Strategic Plan for the College along with the council, to ensure continuity and harmony in projects undertaken annually, with core strategies and action plans. This I consider a great asset in my tenure this year as the proposed committee structure will help in the implementation of the intended projects.

The Ceylon College of Physicians boasts a proud history with a galaxy of eminent physicians who have either revolutionized or added steadily to the field of Medicine in Sri Lanka.

Looking at the panel of past presidents one is struck by their magnanimous contributions from the establishment and development in the fields of Cardiology and Neurology to Postgraduate education, renal transplantation and research.

I'm indeed honored and privileged to felicitate five senior most past presidents, many of whom have been my respected teachers, at today's presidential induction.

Respiratory medicine has progressed slowly over the years in Sri Lanka with the dedication of many committed physicians. The spectrum of respiratory disease has expanded demanding more interventional and therapeutic measures in keeping with the advances. I have had the good fortune to be a part of Sri Lanka's first successful Lung transplantation team last year with Japanese collaboration in the private sector. It is my fervent hope that during my tenure the College could facilitate a Lung Transplantation Program together with the College of Pulmonologists and the Thoracic and vascular surgical teams. Sri Lanka has a well-established Renal transplant program and successful liver transplant program in which the postoperative immune-suppressive regimens are similar. We have today, in the country, trained surgical teams who possess the skill and expertise to progress further. Despite the initial costs this program would be of great benefit for many young patients dying from lung diseases such as pulmonary fibrosis.

\section{Infrastructure}

It is my conviction that the infrastructure of the Ceylon College of Physicians, must be enhanced and strengthened to meet its high expectations. The Royal College of Physicians UK is of Royal Charter since the year 1518 and is placed in the most prestigious location in London. The location of the Ceylon College of Physicians is away from the main hub of medical activities. The finer specialties too are scattered spatially posing many difficulties in coordination and are not easily accessible to most physicians and trainees. This has greatly hampered the participation of many in College activities and training programs. It is our hope that College along with the finer specialties will relocate to a common building, in close proximity to the National Hospital of Sri Lanka.

\section{Qualities of a Physician}

Ladies and Gentleman - The primary focus of the Physician is the patient. The greatest reward that a physician can experience is undoubtedly to see a patient cured. However, this is not always possible, and relief and comfort in suffering is sometimes more important especially with the terminal patient. This is what makes Medicine a vocation not akin to any other profession. Regrettably today the heavy economic demands, academic achievements, in the complex and highly materialistic world have burdened the modern physician overshadowing the true goodness of many. It is a high priority of the College to assist its membership to maintain the nobility of our profession amidst the tremendous pressures of the modern society and critics.

Unity in diversity would be a great strength in this endeavor. I would conclude my address with the reminder that together with professional competence, kindness, compassion and respect to all fellow beings are the greatest qualities that the physician should possess and cultivate to serve mankind with dignity.

I consider it a privilege and a great blessing, to be a son of Sri Lanka, which nurtured me and gave me the luxury of a free education including a post graduate training, despite her meager financial resources, making me what I am today. I do hope that I have repaid, even in part the enormous debt I owe my country. In my capacity as the President of the Ceylon College of Physicians I hope that I with my esteemed council would be able to serve you better and improve the clinical care to be the best in the region. 